\title{
Número especial: Investigación en economía y gestión del deporte
}

\author{
Pablo Gálvez-Ruiz ${ }^{1}(\mathbb{D})$, Jesús Fernández-Gavira² ${ }^{[D}$, Jerónimo García-Fernández² (iD, \\ Jaume García-Villar ${ }^{3}$ (D) \\ ${ }^{1}$ International University of La Rioja (Spain) \\ ${ }^{2}$ University of Seville (Spain) \\ ${ }^{3}$ Universitat Pompen Fabra (Spain)
}

pablo.galvez@unir.net,jesusfgavira@us.es,jeronimo@us.es,jaume.garcia@upf.edu

Received April, 2016

Accepted June, 2016

\section{Resumen}

El deporte supone actualmente uno de los fenómenos sociales de mayor arraigo, el cual además cuenta con una elevada capacidad de movilización. Además posee una gran importancia social así como una importante dimensión económica que si bien son difíciles de cuantificar, se consideran un motor de desarrollo, que implican indirectamente a un gran número de diferentes actividades entre las que se incluyen otras industrias relacionadas con el sector, en las que el deporte constituye un eje vertebrador fundamental.

En este contexto, la Revista Intangible Capital lanza este monográfico en el que se incluye la investigación de la economía del deporte desde diversas perspectivas, destacando así la importancia y el impacto que pueden llegar a tener en diferentes áreas. Por lo tanto, el objetivo de este trabajo es avanzar en el estudio de la economía del deporte a partir de la sinergia de recopilar artículos que traten esta temática.

Palabras clave: Economía, gestión, deporte

Códigos JEL: Z2 
Title: Special Issue: Research in economics and sport management

\section{Abstract}

The sport currently represents one of the most deeply rooted social phenomena, which also have a high capacity for mobilization. It has a social significance and also an important economic dimension that is difficult to quantify accurately, but is considered an engine of development, which imply indirectly to a large number of different activities that include other industries, taking in this regard an important crosscutting.

In this context, Intangible Capital Journal launches this monograph in which research from diverse perspectives of the economy of sport is included, thus highlighting the importance and impact it has on different sectors are integrated. Therefore, the objective of this issue is to advance in the study of sports economics by joining forces and bringing new products through research.

Keywords: Economy, management, sport

Jel Codes: Z2

La extraordinaria trascendencia social que actualmente ostenta el deporte alberga paralelamente una importante dimensión económica que resulta compleja de cuantificar con exactitud, pero que se considera motor de desarrollo al implicar de manera indirecta a un elevado número de actividades distintas que engloban a otras industrias, contando en este sentido con un importante carácter transversal.

De esta manera, en el presente monográfico se integran investigaciones muy diversas donde se aborda la economía del deporte desde diferentes perspectivas implicadas en ella, destacando así la importancia y repercusión que tiene en distintos sectores. En definitiva, el objetivo de este número es avanzar en el estudio de la economía del deporte aunando esfuerzos y aportando novedades a través de sus investigaciones. 
Así, en primer lugar encontramos un estudio de González, Huertas, Valantine, Pérez, Aguado, Calabuig y Crespo, que habla del nivel de emprendimiento empresarial de los estudiantes de Ciencias de la Actividad Física y el Deporte en función de su formación y el género. Utiliza distintas herramientas para valorar la percepción sobre la intención de emprender, el control percibido, las capacidades de emprender, la actitud hacia la conducta, la norma subjetiva, la satisfacción con la vida y el optimismo. En la línea de la percepción de los usuarios, enfoque muy utilizado en el ámbito del deporte dentro del análisis del comportamiento de los consumidores, se encuentra la aportación realizada por Angosto, López y Díaz sobre una muestra de más de 700 participantes en dos ediciones de la misma carrera popular de media distancia, con el objetivo de evaluar las dimensiones comunicación, interacción personal, infraestructura logística y servicios complementarios, de cara a una mejora futura sobre la calidad prestada por la organización.

El estudio que aportan Baños y Rodríguez está enfocado hacia el impacto económico de los eventos deportivos, en este caso orientado al Campeonato del Mundo de Escalada desarrollado en 2014, donde analizan el impacto económico de los no residentes que asistieron al evento, así como el impacto de los gastos realizados por la organización de la competición y que se recuperan en términos de valor añadido bruto.

Dentro del sector low-cost dentro del mercado del fitness en España, se incluye un interesante estudio de Pastor, Prado y Bustillo que analiza la interacción en las clases de actividades dirigidas en un centro de fitness, aportando unos resultados novedosos tras la validación de un instrumento específico para tal fin que consideramos pueden resultar de gran interés para un sector en auge al conocer más aspectos que influyen sobre el servicio.

Por último, encontramos el trabajo aportado por García y Pradas, donde centrándose en el ámbito del golf en Andalucía, analizan el grado de transparencia de las páginas Web de un total de 92 organizaciones inscritas en la Real Federación de Golf de Andalucía. Para ello, diseñan un cuestionario ad hoc compuesto por 4 dimensiones y 11 ítems, obteniendo un alto grado de transparencia y llegando a la conclusión de que la página Web supone un importante elemento para cuantificar el rendimiento del campo sin necesidad de invertir en exceso en otros canales de promoción.

De esta forma, consideramos que las aportaciones recogidas en el presente número contribuyen al entendimiento del impacto de la economía del deporte, siendo fiel reflejo de la realidad interdisciplinar que la caracteriza, coincidiendo la mayoría de autores en la necesidad de continuar avanzando e 
investigando, dada la cantidad de futuras líneas que surgen dentro de cada uno de los trabajos expuestos.

Intangible Capital, 2016 (www.intangiblecapital.org)

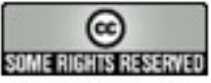

Article's contents are provided on an Attribution-Non Commercial 3.0 Creative commons license. Readers are allowed to copy, distribute and communicate article's contents, provided the author's and Intangible Capital's names are included. It must not be used for commercial purposes. To see the complete license contents, please visit http://creativecommons.org/licenses/by-nc/3.0/. 